\title{
Recent Progress on VLF Wave and Its Interactions with Energetic Particles in the Magnetosphere
}

\author{
Anil Kumar \\ Department of Applied Science (Physics), Bharati Vidyapeeth's College of Engg. Paschim Vihar, New Delhi- \\ 110063(India).
}

\begin{abstract}
Very Low Frequency (VLF) wave is the plasma wave in the magnetosphere with frequency range of 3 $\mathrm{kHz}$ to $30 \mathrm{kHz}$. The universal distribution properties of Very Low Frequency wave (VLF) in the magnetosphere and its interactions with energetic particles, such as the wave-particle resonance, modulation, and particle acceleration, are active topics in space physics research. These problems are fundamentally important issues to understand the energy transport from the solar wind into the magnetosphere. In this paper we briefly reviewed the recent research progress on VLF wave and its interactions with energetic particles in the inner magnetosphere; furthermore, we suggested some open questions for future study.
\end{abstract}

Keywords: Pitch angle diffusion, Fokker-Planck equation, Chorus, Hiss.

\section{Introduction}

Experimental and theoretical studies have shown that radiation belts dynamics display a substantial variability during different levels of geomagnetic disturbances, which is associated with a number of different processes. During the main phase of storms (usually lasting for a few hours), the flux of energetic electrons can decrease by two or three orders of magnitude in the outer radiation belt $3<L<7$. During the recovery phase of a storm, fluxes can increase by a factor of 10-103 over a period of hours to days, with a peak building up near location $L=4$. Such substantial variations in flux are considered as the competition between two processes: the pitch angle scattering process primarily by the Electromagnetic ion cyclotron and ELF hiss waves; and the stochastic acceleration process mainly by the VLF chorus wave, together with inward radial diffusion through drift resonance with enhanced ULF waves. During geomagnetic storms, $\sim \mathrm{MeV}$ electron fluxes can also be enhanced with peaks at pitch angle $90^{\circ}$ by the magnetosonic impulse launched by an interplanetary shock compression of the magnetopause. During geomagnetically quiet conditions, resonant interaction with ELF hiss provides the primary scattering process at location $L>2.5$, leading to losses over tens of days at energies $\sim 1 \mathrm{MeV}$. At lower $L$-values, resonant interactions with lightning-generated whistlers and man-made VLF transmissions dominate the pitch angle scattering process.

\section{Wave-particle Scattering}

Whistler-mode chorus emissions are observed outside the plasmasphere over a broad range of local times (2200- 1300 MLT) with typical frequencies in the range $0.05-0.8 \Omega_{e}$, where $\Omega_{e}$ is the electron gyrofrequency [Tsurutani and Smith, 1974, 1977; Koons and Roeder, 1990; Meredith et al., 2001; Santolik et al., 2003, 2004]. VLF chorus occurs in two frequency bands, a lower band $\left(0.1-0.5 \Omega_{e}\right)$ and an upper band (0.5-1.0 jWej) [Burtis and Helliwell, 1976; Meredith et al., 2001]. Equatorial chorus (jlj < $15 \mathrm{deg}$ ) can be excited by cyclotron resonance with anisotropic $10-100 \mathrm{keV}$ electrons injected near midnight from the plasmasheet [Kennel and Petschek, 1966]. High-latitude chorus (jlj > $15 \mathrm{deg}$ ) may be generated in the horns of the magnetosphere [Meredith et al., 2001]. Santolik et al. [2005] find that equatorial chorus in the upper band extends to about $\mathrm{L}=8$, and in the lower band (below $0.4 \mathrm{jWej}$ ) to $\mathrm{L}=11-12$. Typical chorus amplitudes are $1-$ 100 pT [Burtis and Helliwell, 1975; Meredith et al., 2003a], though amplitudes up to $1 \mathrm{nT}$ have been reported during intense geomagnetic activity [Parrot and Gaye, 1994]. Chorus emissions are predominantly substorm dependent, and all chorus emissions intensify when substorm activity is enhanced [Meredith et al., 2001]. Waveparticle interactions occur when multiples of gyrofrequency equal the wave frequency in the electron reference frame, and this can be expressed in the lab frame by

$\omega-v|| k|=-n| \Omega e \mid / \gamma, n= \pm 1, \pm 2, \ldots(1)$

Here $\mathrm{v} \|=\mathrm{v} \cos \alpha$ with $\mathrm{v}$ being the velocity and $\alpha$ being the pitch angle, $\gamma$ is the resonant relativistic Lorentz factor. Figure 1 presents a schematic graph of the possible wave-particle interactions associated with energetic electrons in the Earth's inner magnetosphere. The dotted black lines represent the power flux density in each wave's frequency range (left $Y$-axis). The three long narrow regions represent the gyrating, bouncing, and drifting frequency in different $L$-shells for electrons with different resonant energy (right $Y$-axis). 

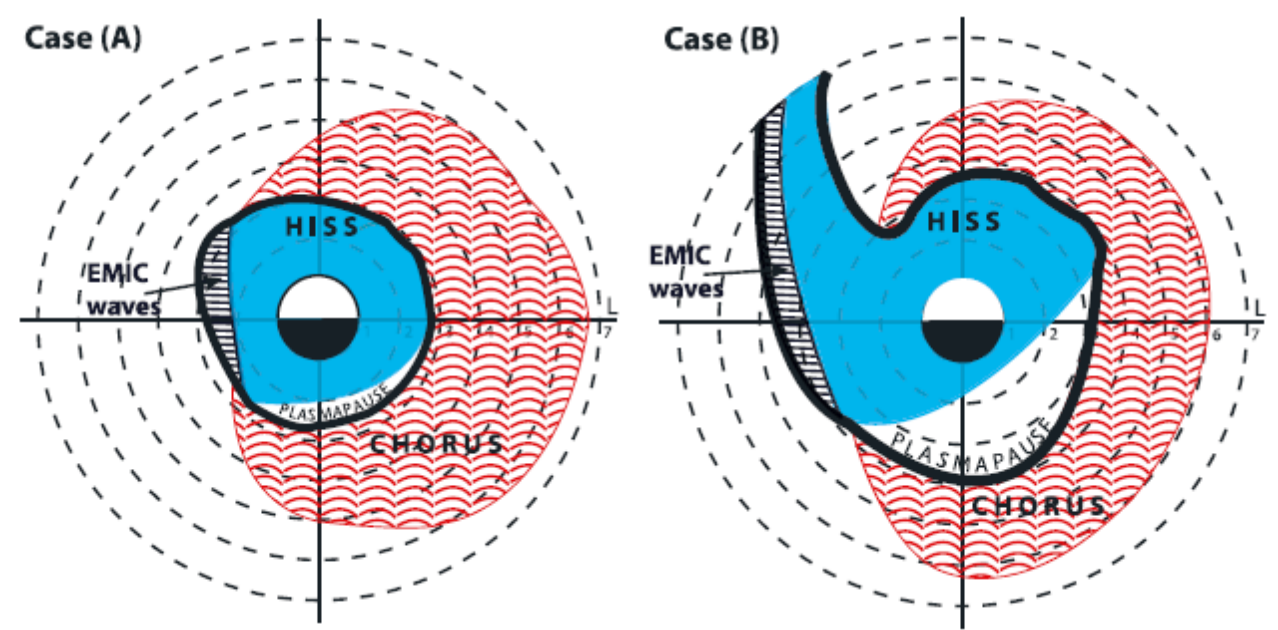

Figure 1. Schematic distributions of chorus, plasmaspheric hiss, and EMIC waves in the cases of high geomagnetic activity and relatively compressed plasmasphere (case A) and following high geomagnetic activity during the evolution of a plasmaspheric plume (case B) during the main phase (A) and recovery phase (B) of a storm.

Wave-particle interactions lead to the violation of the first and second adiabatic invariants and diffusion of electrons in pitch-angle and energy. Pitch-angle diffusion scatters electrons into the loss cone and produces precipitation of electrons, whereas energy diffusion can generate acceleration of electrons and harden the energy spectrum. To evaluate these processes, a solution of 2-D bounce-averaged Fokker-Planck equation describing the local acceleration and loss processes, together with incorporation of detailed information of the amplitudes and spectral properties of the waves is required. Such diffusion equation associated with the phase space density $\sigma$ can be written by

$$
\begin{aligned}
& \frac{\partial \sigma}{\partial t}=\frac{1}{G p} \frac{\partial}{\partial \alpha_{e}}\left[G\left(\hat{D}_{\alpha \alpha} \frac{1}{p} \frac{\partial \sigma}{\partial \alpha_{e}}+\hat{D}_{\alpha p} \frac{\partial \sigma}{\partial p}\right)\right] \\
& +\frac{1}{G} \frac{\partial \sigma}{\partial p}\left[G\left(\hat{D}_{p \alpha} \frac{1}{p} \frac{\partial \sigma}{\partial \alpha_{e}}+\hat{D}_{p p} \frac{\partial \sigma}{\partial p}\right)\right]
\end{aligned}
$$

Here $p$ is the particle's momentum, $G=p^{2} T(\alpha e) \sin \alpha e \cos \alpha e$ with $\alpha e$ being the equatorial pitch angle, $T(\alpha e)$ $\approx 1.30-0.56 \sin \alpha e$ gives the normalized bounce time; $D \alpha \alpha, D p p$, and $D \alpha p=D p \alpha$ represent bounce-averaged diffusion coefficients in pitch angle, momentum and cross pitch angle-momentum.

\section{Method of Calculation of Pitch angle diffusion}

We assume infinite, homogeneous, collisionless plasma immersed in a uniform, static magnetic field $B_{0}=B_{0} e_{z}$, in the presence of superposed electromagnetic waves [Inan, 1977]. We use quasi-linear diffusion theory to describe the effects of the waves on the particles in terms of a kinetic equation for the gyrophase-averaged phase-space density $\Phi$. Ensemble averaging of the wave fields is carried out. The general, relativistic quasi-linear diffusion equation for $\Phi$, in the limit of gyroresonant diffusion, can be written in the form [e.g., Melrose, 1980], Summers et al (2007).

$\frac{\partial \Phi}{\partial t}=\frac{1}{\sin \alpha} \frac{\partial}{\partial \alpha}\left(D_{\alpha \alpha} \sin \alpha \frac{\partial \Phi}{\partial \alpha}\right)+\frac{1}{\sin \alpha} \frac{\partial}{\partial \alpha}\left(D_{\alpha p} \sin \alpha \frac{\partial \Phi}{\partial p}\right)+\frac{1}{p^{2}} \frac{\partial}{\partial p}\left(p^{2} D_{p \alpha} \frac{\partial \Phi}{\partial \alpha}\right)+\frac{1}{p^{2}} \frac{\partial}{\partial p}\left(p^{2} D_{p p} \frac{\partial \Phi}{\partial p}\right)$

where $D_{\alpha \alpha}, D_{\alpha p}=D_{p \alpha}$ and $D_{p p}$ are the diffusion or Fokker-Planck coefficients which depend on the properties of the waves; $p=\gamma m_{\sigma} v$ is the momentum of the particle of species $\sigma$, rest mass $m_{\sigma}$ and speed v; $\gamma=\left(1-\frac{v^{2}}{c^{2}}\right)^{-1 / 2}$ is the Lorentz factor (c is the speed of light); $\alpha$ is the pitch angle of the particle, and $\mathrm{t}$ denotes time. In the present study we treat only the special case of electromagnetic waves propagating parallel or antiparallel to the background magnetic field $B_{0}$. We assume that the R-mode (s=1) and L-mode (s=-1) waves each have the Gaussian spectral density, 


$$
W_{S}(\omega)=\frac{\left|\Delta B_{S}\right|^{2}}{8 \pi} \frac{1}{\rho} \frac{1}{\delta \omega} e^{-\left(\frac{\omega-\omega_{m}}{\omega}\right)^{2}},
$$

with

$$
\rho=\frac{\sqrt{\pi}}{2}\left[\operatorname{erf}\left(\frac{\omega_{m}-\omega_{1}}{\delta \omega}\right)+\operatorname{erf}\left(\frac{\omega_{2}-\omega_{m}}{\delta \omega}\right)\right],
$$

where $\omega_{1}$ is the lower frequency limit, $\omega_{2}$ is the upper frequency limit, $\omega_{m}$ is the frequency of maximum wave power, $\delta \omega$ is a measure of the bandwidth, and erf is the error function. The wave spectral density (2) has been normalized, so that (Summers et al, 2007).

$$
\frac{\left|\Delta B_{S}\right|^{2}}{8 \pi}=\int_{\omega_{1}}^{\omega_{2}} W_{S}(\omega) d \omega
$$

where $\left|\Delta B_{S}\right|^{2}$ is the mean wave amplitude.

Following the study of Summers et al $(2005,2007)$, we can now express the diffusion coefficients for the particle species $\sigma$ as follows:

$$
\begin{aligned}
& D_{\alpha \alpha}=\frac{\pi}{2} \frac{1}{\rho} \frac{\Omega_{\sigma}^{2}}{\left|\Omega_{e}\right|} \frac{1}{(E+1)^{2}} \sum_{s} \sum_{j} \frac{R\left(1-\frac{x_{j} \cos \alpha}{y_{j} \beta}\right)^{2}\left|d x_{j} / d y_{j}\right|}{\delta x\left|\beta \cos \alpha-d x_{j} / d y_{j}\right|} e^{-\left(\frac{x_{j}-x_{m}}{\delta x}\right)^{2}} \\
& \frac{D_{\alpha p}}{p}=\frac{\pi}{2} \frac{1}{\rho} \frac{\Omega_{\sigma}^{2}}{\left|\Omega_{e}\right|} \frac{1}{\beta} \frac{\sin \alpha}{(E+1)^{2}} \sum_{s} \sum_{j} \frac{R\left(\frac{x_{j}}{y_{j}}\right)\left(1-\frac{x_{j} \cos \alpha}{y_{j} \beta}\right)^{2}\left|d x_{j} / d y_{j}\right|}{\delta x\left|\beta \cos \alpha-d x_{j} / d y_{j}\right|} e^{-\left(\frac{x_{j}-x_{m}}{\delta x}\right)^{2}}
\end{aligned}
$$

where we have introduced the dimensionless variables,

$$
x=\frac{\omega}{\left|\Omega_{e}\right|}, \quad y=\frac{c k}{\left|\Omega_{e}\right|}
$$

and $\mathrm{E}$ is the dimensionless particle kinetic energy given by

$$
E=E_{k} /\left(m_{\sigma} c^{2}\right)=\gamma-1 ; \beta=\frac{v}{c}=[E(E+2)]^{1 / 2} /(E+1) ;\left|\Omega_{e}\right|=e\left|B_{0}\right| /\left(m_{e} c\right)
$$

is the nonrelativistic electron gyrofrequency, where $e$ is the unit charge; $\left|\Omega_{\sigma}\right|=q\left|B_{0}\right| /\left(m_{\sigma} c\right)$ is the nonrelativistic particle gyrofrequency, where $q$ is the particle charge; $R=\left|\Delta B_{S}\right|^{2} / B_{0}^{2}$ is the ratio of the energy density of the turbulent magnetic field to that of the background field, i.e., the relative wave power; $x_{m}=\frac{\omega_{m}}{\left|\Omega_{e}\right|}, \delta x=\frac{\delta \omega}{\left|\Omega_{e}\right|}$; and the derivative $d x_{j} / d y_{j}=d x\left(y_{j}\right) / d y$ is determined from the appropriate dispersion relation.

\section{Variation with Frequency:}

\section{Results and Discussion}

Figure (1) shows variation of pitch angle diffusion coefficient $\left[\mathrm{D} \alpha \alpha\left(\mathrm{J}^{* *} / \mathrm{S}\right)\right]$ with interacting frequencies at different L-values. Three parallel lines in this figure are corresponding to $\mathrm{L}=1.3,1.5 \& 1.7$ at VLF frequencies $3 \mathrm{kHz} ., 4 \mathrm{kHz} \& 5 \mathrm{kHz}$. respectively. There is also an exponential variation between [D $\alpha \alpha(\mathrm{J} * * / \mathrm{S})]$ \& VLF frequencies. We see that as interacting frequencies increases pitch angle diffusion coefficient of particles also increases. Moreover this variation is not only interacting frequencies dependent but also L-values dependent; we see that pitch angle diffusion coefficient is low for all interacting frequency ranges for $\mathrm{L}=1.3$ and 
it is high for $\mathrm{L}=1.7$. It is in agreement with works done by various author in India (Singh DP) as well as Stanford University.

There are peculiar situations that pitch angle diffusion coefficient increases with interacting frequency linearly, i.e. D $\alpha \alpha$ and frequency graph is almost a straight line. Such things appear for all L-values. We can conclude that large frequency and large L-values takes effective role in the diffusion of electrons into loss cone. As we have said this is an agreement with works of large number of whistler mode wave worker. In this case the wave-width value taken to be $50 \mathrm{~Hz}$. and wave magnetic field $\mathrm{Bw}=0.001 \mathrm{nT}$ and the pitch angle has taken to be $50^{0}$.

It is clear that at low L-shells, diffusion is weak and at high latitude it increases from moderate to strong diffusion (Singh 1991).

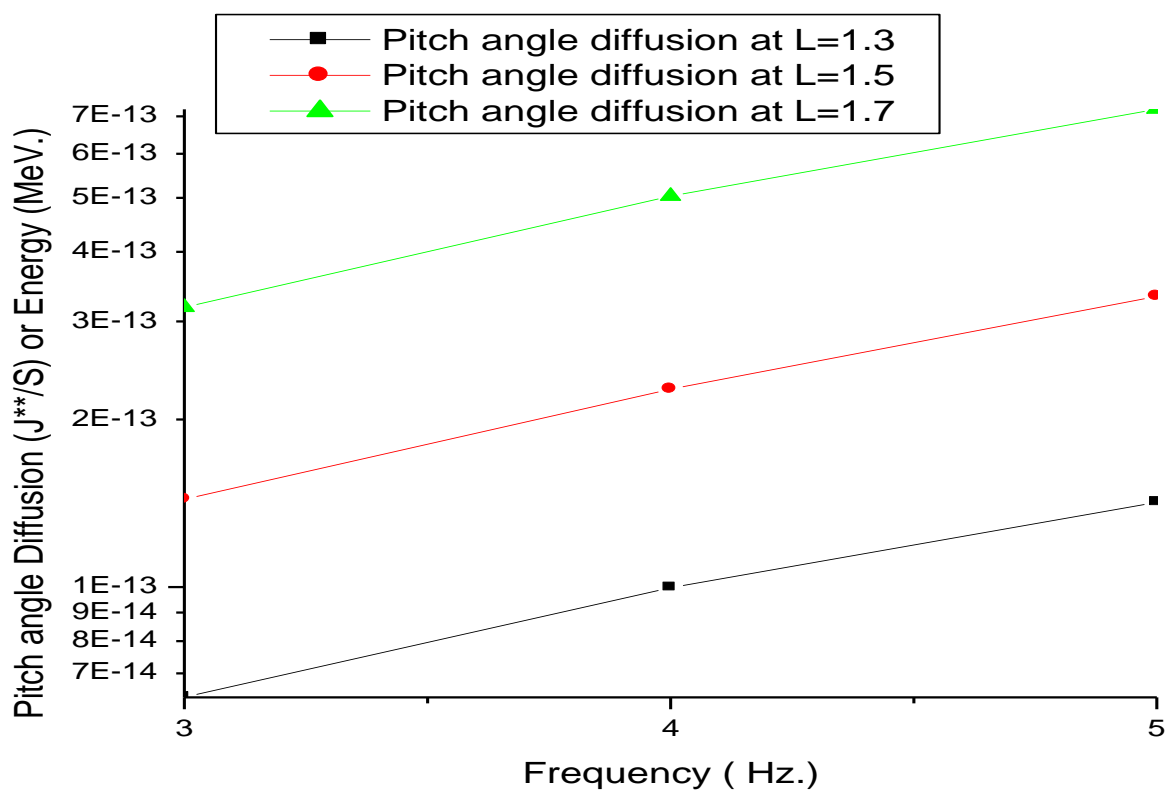

Fig. 1: Variation of pitch angle diffusion coefficient $\mathrm{D} \alpha \alpha\left(\mathrm{J}^{2} / \mathrm{s}\right)$ with interacting frequencies at different LValues, Keeping $\Delta \mathrm{F}=50 \mathrm{~Hz}$., $\mathrm{Bw}=0.001(\mathrm{nT})$ and pitch angle $50^{\circ}$.

\section{Variation With L - Values:}

Figure (2) shows variation of energy diffusion coefficient [Dpp (J**/S)] with L-values at different wave magnetic field. Three parallel lines in this figure are corresponding to $\mathrm{Bw}=0.001,0.01 \& 0.1$ (nT) respectively. There is also an exponential variation between Dpp \& L-values. We see that as L-values increases energy diffusion coefficient of particles also increases. Moreover this variation is not only L-values dependent but also wave magnetic field $(\mathrm{Bw})$ dependent; we see that energy diffusion coefficient is low for all L-value ranges for $\mathrm{Bw}=0.001(\mathrm{nT})$ and it is high for $\mathrm{Bw}=0.1(\mathrm{nT})$. It is in agreement with works done by various author in India (Singh DP) as well as Stanford University.

There is a peculiar situation that energy diffusion coefficient increases with L-value linearly, i.e. Dpp and L-values graph is almost a straight line. Such things appear for all wave magnetic field (Bw). We can conclude that large wave magnetic field $(\mathrm{Bw})$ and large L-values takes effective role in the diffusion of electrons into loss cone. As we have said this is an agreement with works of large number of whistler mode wave worker. In this case the wave-width value taken to be $50 \mathrm{~Hz}$. and interacting frequency $=3 \mathrm{kHz}$, the pitch angle has taken to be $50^{\circ}$. There exist various magetohydrodynamic waves with different frequencies and other properties, such as ULF wave, Very Low Frequency wave (VLF), Electromagnetic Ion Cyclotron wave (EMIC), etc. If the corresponding resonance conditions are satisfied, there can be wave-particle interaction between these waves and energetic particles in inner magnetosphere, causing the damping (amplifying) of the wave and acceleration (deceleration) of the particles. 


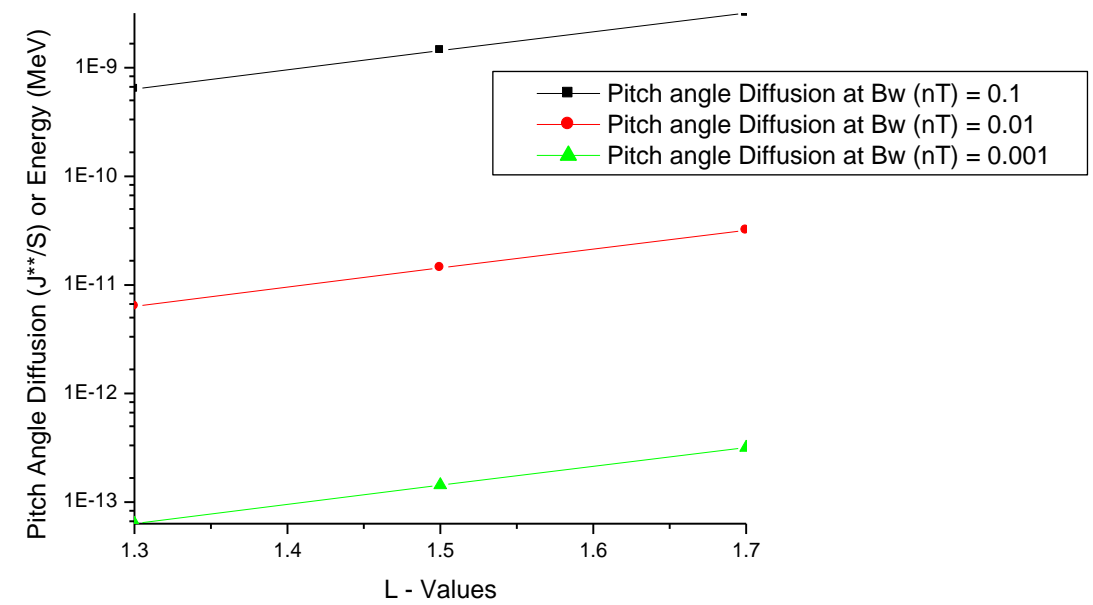

Fig. 2: Variation of energy diffusion coefficient $\operatorname{Dpp}\left(\mathrm{J}^{2} / \mathrm{s}\right)$ with L-values at different Bw (nT), Keeping interacting frequency $=3 \mathrm{kHz}$., $\Delta \mathrm{F}=50 \mathrm{~Hz}$. and pitch angle $50^{\circ}$.

\section{Conclusions}

The current numerical simulations in this paper demonstrate that resonant interactions with VLF/ELF waves play crucial roles in the dynamics of the inner magnetosphere. Pitch angle scattering of radiation belt electrons will produce electron losses, while energy diffusion can produce acceleration comparable to or stronger than losses. Diffusion with respect to any of the variables (pitch angle, energy, or L) will change the gradients with respect to two other variables. For example, pitch angle scattering at lower L shells may increase radial gradients and increase inward radial diffusive transport. If waves that produce pitch angle scattering are in resonance with electrons only for a limited range of energies, pitch angle diffusion will also change energy gradients and will affect energy diffusion. Radial diffusion will change the energy spectrum by accelerating or decelerating electrons and will also change the pitch angle distribution. While this paper provides an initial assessment of the dominant acceleration and loss processes.

\section{Acknowledgments}

Author is very thankful to BVCOE, New Delhi principal for providing his support during experimental work done in the college physics lab.

\section{References}

[1]. Burtis W J, Helliwell R A. Magnetospheric chorus: Occurrence patterns and normalized frequency. Planet Space Sci, 1976, 24: 1007

[2]. Green J C, Kivelson M G. Relativistic electrons in the outer radiation belt: Differentiating between acceleration mechanisms. J GeophysRes, 2004, 109: A03213.

[3]. Fok, M.-C., R. B. Horne, N. P. Meredith, and S. A. Glauert (2008), Radiation Belt Environment model: Application to space weather nowcasting, J. Geophys. Res., 113, A03S08, doi:10.1029/2007JA012558.

[4]. Horne, R. B., and R. M. Thorne (1998), Potential wave modes for electron scattering and stochastic acceleration to relativistic energies during magnetic storms, Geophys. Res. Lett., 25, 3011 - 3014, doi:10.1029/98GL01002.

[5]. Horne, R. B., et al. (2005a), Wave acceleration of electrons in the Van Allen radiation belts, Nature, 437, 227-230, doi:10.1038/nature03939.

[6]. Hudson, M. K., S. R. Elkington, J. G. Lyon, C. C. Goodrich, and T. J. Rosenberg (1999), Simulation of radiation belt dynamics driven by solar wind variations, in Sun-Earth Plasma Connections, Geophys. Monogr. Ser., vol. 109, edited by J. L. Burch, R. L. Carovillano, and S. K. Antiochos, pp. 171-182, AGU, Washington, D. C.

[7]. Inan US, Non-linear Gyro-resonant interactions of energetic particles and coherent VLF waves in the magnetosphere, Tech. Rept. No. 3414-3 Stanford Univ., Stanford, CA.1977.

[8]. Jordanova, V. K., L. M. Kistler, J. U. Kozyra, G. V. Khazanov, and A. F. Nagy (1996), Collisional losses of ring current ions, J. Geophys. Res., 101, 111 - 126, doi:10.1029/95JA02000.

[9]. Kennel, C. F., and F. Engelmann (1966), Velocity space diffusion from weak plasma turbulence in a magnetic field, Phys. Fluids, 9 , 2377, doi:10.1063/1.1761629.

[10]. Parrot M, Gaye C A. A statistical survey of ELF waves in a geostationary orbit. Geophys Res Lett, 1994, 21: 2463-2466.

[11]. Santolik O, Macusova E, Yearby K H, et al. Radial variation of whistler-mode chorus: First results from the STAFF/DWP instrument on board the Double Star TC-1 spacecraft. AnnGeophys, 2005, 23:2937.

[12]. Shprits, Y. Y., and R. M. Thorne (2004), Time-dependent radial diffusion modeling of relativistic electrons with realistic loss rates, Geophys. Res. Lett., 31, L08805, doi:10.1029/2004GL019591.

[13]. Shprits, Y. Y., R. M. Thorne, R. Friedel, G. D. Reeves, J. Fennell, D. N. Baker, and S. G. Kanekal (2006b), Outward radial diffusion driven by losses at magnetopause, J. Geophys. Res., 111, A11214, doi:10.1029/2006JA011657. 
[14]. Summers, D., R. M. Thorne, and F. Xiao (1998), Relativistic theory of wave-particle resonant diffusion with application to electron acceleration in the magnetosphere, J. Geophys. Res., 103, 20,487 - 20,500, doi:10.1029/98JA01740.

[15]. Summers, D., B. Ni, and N. P. Meredith (2007), Timescales for radiation belt electron acceleration and loss due to resonant waveparticle interactions: 2. Evaluation for VLF chorus, ELF hiss, and electromagnetic ion cyclotron waves, J. Geophys. Res., 112, A04207, doi: 10.1029/ 2006JA011993.

[16]. Singh DP, strong diffusion of resonant electrons by VLF waves, Indian J Radio and Space Phys, 20 (1991) 424. 